\title{
Self Duality in Super Yang-Mills Theories
}

\author{
C. R. Gilson, I. Martin, A. Restuccia and J. G. Taylor \\ Department of Mathematics, King's College, London WC2 2LS, United Kingdom
}

\begin{abstract}
The self dual condition in superspace is analysed for $N=1,2,4$ super Yang-Mills theories. A complete solution of all the constraints in terms of a light cone superfield $J$ is presented, where the only equation that $J$ satisfies is a SUSY generalization of the Yang equation. By reduction of that equation we obtain various two dimensional SUSY models. We introduce the associated linear problem in terms of $J$, whose integrability condition gives us back the super Yang equation and allows us to obtain the Kac-Moody algebra structure of the theory.
\end{abstract}

\section{Introduction}

Self-duality conditions and self-dual Yang-Mills fields have been studied extensively in relation to the non-perturbative approach to quantum field theory. They provide finite action solutions to the field equations, e.g. instantons and in lower dimensions, monopoles and solitons. The solvability of these non-linear systems is related to the existence of a linear problem whose integrability condition provides the original non-linear equations. This problem has been extensively analysed in the literature, also in relation to other mathematical properties such as the Painlevé property and the associated infinite-dimensional symmetries [1,2,3]. Recently, a great deal of interest has been given to the supersymmetric extension of Yang-Mills and gravity from the unification point of view and the finiteness of the associated quantum theories, in particular $N=4$ super Yang-Mills. Consequently, it is important to analyse the supersymmetric extension of the self-dual Yang-Mills fields $[4,5,6]$ and the lower dimensional reductions like SUSY chiral theories, SUSY Sine Gordon, which have already been considered [7]. The linear problem associated with SUSY Yang-Mills has been tackled by Volovich [8] and used by Devchand and L.-L. Chau [9] to analyse the infinite symmetries of the field equations. However, the solvability of these non-linear systems has not yet been proved, so that a detailed analysis in terms of the component fields is therefore in order. A first step towards the solution of this problem is to consider the super self dual Yang-Mills (SDSYM) equations and their relation to the dimensionally reduced supersymmetric versions. In Sect. 2, we discuss SDSYM in the most general 
framework, including theories with central charges. In Sect. 3 we solve explicitly the SDSYM conditions in terms of a light cone superfield which satisfies the supersymmetric analog of the Yang equation. In Sect. 4, we look at possible reductions to lower dimensions and compare them with the known supersymmetric version already studied. Finally, by analogy with SDSYM we can obtain the linear problem associated to the SDSYM equations, and from it, using the H-Transform method, we can analyse the group structure of the infinite parameter symmetry in the various theories under discussion.

\section{Section 2. Self Duality in Superspace}

In this and the next section we consider the self dual supersymmetric $[9,10,11]$ Yang-Mills (SDSYM) equations. We obtain a formulation in terms of a light cone superfield $J(x, \theta, \bar{\theta})$ which is a natural generalization of the $J$-formulation of self-dual Yang-Mills (SDYM) $[4,5,12]$ equations. The formulation is therefore manifestly light cone supersymmetric. It is then straightforward to deduce from this the superfield linear problem whose integrability conditions yield the SDSYM equations. The Kac-Moody algebra follows directly from this linear problem.

We take $\left(x^{a}, \theta^{\alpha}, \bar{\theta}^{\dot{\alpha}}\right)$ as the coordinates of the grassmannian supermanifold, $x^{a}$ are the even coordinates $\theta^{\underline{\alpha}} \equiv \theta_{i}^{\alpha}$ the odd ones. The index $i$ represents the number of supersymmetric generators realized in the theory.

We may work in Euclidean superspace. In that case $\theta^{\underline{\alpha}}$ and $\bar{\theta}^{\underline{\alpha}}$ are not related by complex conjugation as in a Lorentz superspace. In Euclidean superspace one may however use hermiticity under the unitary involutory operator of Osterwalder and Schrader [13]. The superfields are then expressed only in terms of $\theta^{\alpha}$ and $\bar{\theta}^{\alpha}$ as one has for the Lorentz superfields. We may instead consider the Lorentz superspace. In that case one may impose a self duality condition provided that one considers a complex gauge vector for the $\mathrm{Y}-\mathrm{M}$ potential [11]. In terms of the geometrical objects that means one considers complex superfields. We follow this approach here. It is clear how to obtain the corresponding results in the Euclidean superspace $[14,24]$. The self duality condition for SYM was first considered in [25] and more recently in terms of supercurvatures in $[10,11]$.

We define the self duality condition for SYM as

$$
{ }^{*} F_{a b}=\frac{1}{2} \varepsilon_{a b c d} F^{c d}=i F_{a b},
$$

where $F_{a b}$ is the supercurvature tensor. This condition, together with the supercurvature constraints which define the SYM multiplet determine a self dual on-shell multiplet. Clearly the $\theta=\bar{\theta}=0$ component of $(1)$ is the self dual condition for the helicity 1 field component. This condition implies the field equation for that field and by sypersymmetry it also implies the field equation for all the other components of the supermultiplet.

The constraints which isolate the $N=1 \mathrm{SYM}$ multiplet [15] are

$$
\begin{aligned}
& F_{\alpha \beta}=0, \\
& F_{\dot{\alpha} \hat{\beta}}=0, \\
& F_{\alpha \hat{\beta}}=0 .
\end{aligned}
$$


All the curvatures are given in terms of the chiral superfields $W^{\underline{\alpha}}$ and $\bar{W}^{\underline{\alpha}}$.

The constraints for $N=2$ SYM [16] are

$$
F_{\alpha \beta}^{(i j)}=0, \quad F_{\alpha \beta}^{(i j)}=0, \quad F_{\alpha \beta_{J}}^{l}=0, \quad i=1,2 .
$$

All the curvatures are given in terms of the chiral superfields, $W$ and $\bar{W}$.

The constraints of $N=4$ SYM [17] are the same as (3), but now $i=1,2,3,4$. The curvatures are all given in terms of 6-plets of SU(4) $W^{i j}$ and $\bar{W}^{i j}$. For $N=1$ and $N=2$ SYM the supermultiplets defined by (2) and (3) are off-shell, while for $N=4$ SYM they yield field equations. We may also consider a central charge extension of the supersymmetric (SUSY) algebra $[18,19,20]$. In this case one realizes the algebra in a superspace with additional bosonic coordinates $z^{i j}$. In particular if we consider only one central charge generator, the superspace now has coordinates $\left(x^{a}, \theta^{\alpha}, \bar{\theta}^{\dot{\alpha}}, z\right)$. In this case there are additional constraints to isolate the off-shell SYM multiplet.

For $N=2$ SYM with one central charge [19] in addition to (3) we must impose

$$
\begin{aligned}
& \mathscr{D}_{\alpha}{ }^{\left(i \mathscr{D}^{\alpha j)}\right.} W=0 \\
& \mathscr{D}_{\dot{\alpha}}{ }^{(i} \mathscr{D}^{\dot{\alpha} j)} \bar{W}=0 .
\end{aligned}
$$

The internal indices $i$ and $j$ are raised and lowered with USp(2) metric $\varepsilon^{i j}$.

For $N=4$ SYM with one central charge [23] we have to impose in addition to (3) the traceless conditions

$$
\bar{\alpha}_{i j} W_{c}^{i j}=0, \quad \alpha^{i j} \bar{W}_{c i j}=0,
$$

where $\alpha^{i j}$ is the USp (4) metric.

The basic supermultiplet $W_{c}^{i j}$ is now a 5-plet of USp(4). We have relaxed the usual reality condition one imposes in SYM and instead we now impose the self duality condition (1).

Let us consider first the multiplets without central charges. From the Bianchi identities one obtains for $N=1$,

$$
F_{a b}=\frac{1}{32} i\left(\mathscr{D}_{\alpha} \sigma_{a b \beta}^{\alpha} W^{\beta}+\overline{\mathscr{D}}_{\dot{\alpha}} \bar{\sigma}_{a b \dot{\beta}}^{\dot{\alpha}} \bar{W} \dot{\beta},\right.
$$

and similar expressions for $N=2$ and $N=4 \mathrm{SYM}$ in terms of $W, \bar{W}$ and $W^{i j}, \bar{W}_{i j}$ respectively.

The self duality condition (1) then implies

$$
\mathscr{D}_{(\alpha} W_{\beta)}=0 \text {. }
$$

Further use of the constraints (2) yields $\mathscr{D}_{\alpha} W_{\beta}=0$, which together with the chiral character of $W_{\beta}, \mathscr{D}_{\underline{\alpha}} W_{\beta}=0$ imply

$$
W_{\beta}=0 .
$$

An analogous argument shows that

$$
W=0
$$

for $N=2$ SDSYM and

$$
W^{i j}=0
$$

for $N=4$ SDSYM. 
For the multiplets with central charges one may show [23], also by the use of the Bianchi identities, that

$$
F_{a b}=\frac{1}{160}\left\{\bar{\sigma}_{b \gamma}^{\dot{\alpha}} \bar{\sigma}_{a}^{\dot{\gamma} \gamma} \mathscr{D}_{\dot{\alpha} k} \mathscr{D}_{\dot{\gamma} l} \bar{W}_{c}^{l k}+\bar{\sigma}_{b \dot{\gamma}}{ }^{\alpha} \bar{\sigma}_{a}^{\dot{\gamma}} \mathscr{D}_{\alpha}{ }^{k} \mathscr{D}_{\gamma}{ }^{l} W_{c l k}\right\} \text {. }
$$

We consider the $N=4$ case, the $N=2$ gives analogous results.

Again the self duality condition implies

$$
\mathscr{D}_{(\alpha}{ }^{k} \mathscr{D}_{\beta)} W_{c k l}=0 \text {. }
$$

We then consider a partial gauge fixing in the theory, $\mathscr{D}^{\alpha \dot{\alpha}} \partial_{Z} A_{\alpha \dot{\alpha}}=0$.

We are then left with gauge transformations $\delta A_{\underline{\alpha}}=\mathscr{D}_{\alpha} \Lambda$, which are $z$ independent only, $\partial_{z} \Lambda=0$. Moreover $A_{z}$ now transforms as a curvature. We introduce then $W^{i j}=W_{c}^{i j}-2 \alpha^{i j} A_{z}$. It can then be shown that (11) implies $\partial_{z} W^{i j}=0$. The theory is now exactly [23] the $N=4$ SDSYM without central charges, $W^{i j}$ being the 6-plet of $\mathrm{SU}(4)$. We thus regain the same result (10). We have now to analyse the physical content of the other multiplet $\bar{W}_{\dot{\beta}}, \bar{W}$ or $\bar{W}^{i j}$ for $N=1,2$ and 4SYM. One may directly proceed as in ordinary self dual YM theory from (1) by introducing light cone variables in Minkowski space or Yang variables in Euclidean space. One then obtains the usual expressions for the components of $A_{a}$, now in terms of superfields,

$$
\begin{array}{ll}
A_{y}=D^{-1} \partial_{y} D, & A_{+}=D^{-1} \partial_{+} D, \\
A_{\bar{y}}=\bar{D}^{-1} \partial_{\bar{y}} \bar{D}, & A_{-}=\bar{D}^{-1} \partial_{-} \bar{D} .
\end{array}
$$

Defining the superfield $j=D D^{-1}$, we get

$$
\partial_{\bar{y}}\left(j^{-1} \partial_{y} j\right)+\partial_{-}\left(j^{-1} \partial_{+} j\right)=0
$$

This superfield equation is equivalent to several component equations which are not independent. In fact we have to take into account constraints (2) and (3) which relate the different components of $j$.

For example for $N=1$ we notice that by choosing the supersymmetric gauge $A_{\dot{\alpha}}=0$ and using that $F_{a \dot{\alpha}}=0$, which is a consequence of $W_{\beta}=0$ we obtain $D_{\dot{\alpha}} A_{a}=0$. $A_{a}$ is a chiral superpotential in that gauge. This implies the chirality of $j$. However the different components of $j$ are still not independent and we must further analyse (1) and (2) to completely solve this problem. We observe that the $\theta=\bar{\theta}=0$ component of (12) is the Yang equation for the helicity 1 self dual component. We now proceed to explicitly solve the whole set of constraints.

\section{Section 3. $N=1$ The Explicit Solution}

The solution of (2a), flatness in the $\alpha$-grassmannian direction, is $A_{\alpha}=e^{-v} D_{\alpha} e^{v}$. Analogously the solution of (2b) is $A_{\dot{\alpha}}=e^{-u} \bar{D}_{\dot{\alpha}} e^{u}$. (2c) is a conventional constraint, which allows us to obtain $A_{a}$ in terms of $A_{\alpha}$ and $A_{\dot{\alpha}}$.

$V$ and $U$ are complex superfields, in the adjoint representation of the gauge group, which we cannot constrain further from the conditions (2).

After imposing the supersymmetric gauge condition $A_{\dot{\alpha}}=0$, the gauge freedom of the theory reduces to a pre-gauge transformation $e^{v} \rightarrow e^{-\Lambda_{1}} e^{v} e^{\Lambda_{1}}$, where $\Lambda_{1}$ is a chiral superfield $D_{\alpha} \Lambda_{1}=0$, and a gauge transformation with parameter 
$\Lambda_{2}: D_{\dot{\alpha}} \Lambda_{2}=0$. We now proceed by using light-cone coordinates. We denote by $x^{ \pm}=1 / \sqrt{ } 2\left(x^{0} \pm x^{3}\right), x^{i}(i=1,2)$ the even coordinates and by $\theta^{+} \equiv \theta^{1}, \theta^{-} \equiv \theta^{2}$, $\bar{\theta}^{+}=\bar{\theta}^{1}, \bar{\theta}^{-}=\bar{\theta}^{i}$ the odd ones. The light-cone supersymmetric subalgebra has generators $Q_{+\alpha}=\left(\Pi_{+} Q\right)_{\alpha}$ in terms of the original supersymmetric generators $Q_{\alpha}$, where $\Pi_{+}=\frac{1}{2} \gamma_{+} \gamma_{-}$and $\Pi_{-}=\frac{1}{2} \gamma_{-} \gamma_{+}$are the spinor projectors, $\gamma_{+}=1 / \sqrt{ } 2\left(\gamma^{0} \mp \gamma^{3}\right)$.

It is known that SYM can be explicitly expressed in the light cone gauge in terms of light-cone superfields $[21,22]$. The theory is not Lorentz covariant but it is manifestly supersymmetric under the subalgebra generated by $Q_{+\alpha}$.

We may now use the Wess-Zumino gauge as in [21] to obtain

$$
V\left(x^{m}, \theta^{\alpha}, \bar{\theta}^{\alpha}\right)=\theta^{-} \bar{\theta}^{+} \phi\left(x, \theta^{+}\right)+\theta^{+} \bar{\theta}^{-} \bar{\phi}\left(x, \bar{\theta}^{+}\right)+\theta^{-} \bar{\theta}^{-} C\left(x, \theta^{+}, \bar{\theta}^{+}\right),
$$

where $\bar{\theta}^{+}, \bar{\theta}^{-}$are the complex conjugates to $\theta^{+}$and $\theta^{-}, \phi\left(x, \theta^{+}\right)$and $\bar{\phi}\left(x, \bar{\theta}^{+}\right)$are independent superfields, which depend only on the odd variables indicated. In the process we have fixed completely the gauge.

We proceed now to solve (8). It is convenient to introduce the left-handed coordinates $x_{L}{ }^{m}=x^{m}+i \theta \sigma^{m} \bar{\theta}$. We rewrite $V$ as

$$
\begin{aligned}
V\left(x^{m}, \theta^{\alpha}, \bar{\theta}^{\dot{\alpha}}\right) & =V\left(x_{L}{ }^{m}-i \theta \sigma^{m} \bar{\theta}, \theta^{\alpha}, \bar{\theta}^{\dot{\alpha}}\right)=V\left(x_{L}{ }^{m}, \theta^{\alpha}, \bar{\theta}^{\dot{\alpha}}\right)-i \theta \sigma^{m} \bar{\theta} \partial_{m} V\left(x_{L}{ }^{m}, \theta^{\alpha}, \bar{\theta}^{\dot{\alpha}}\right) \\
& =\theta^{-} \bar{\theta}^{+} \phi\left(x_{L}{ }^{m}, \theta^{+}\right)+\theta^{+} \bar{\theta}^{-} \bar{\phi}\left(x_{L}{ }^{m}, \bar{\theta}^{+}\right)+\theta^{-} \bar{\theta}^{-} U\left(x_{L}{ }^{m}, \theta^{+}, \bar{\theta}^{+}\right),
\end{aligned}
$$

where

$$
U\left(x_{L}, \theta^{+}, \bar{\theta}^{+}\right)=C\left(x_{L}, \theta^{+}, \bar{\theta}^{+}\right)+\theta^{+} \bar{\theta}^{+}\left(i \partial \phi+i \bar{\partial} \bar{\phi}-\left(\partial / \partial x^{+}\right) C\right), \partial=\partial /\left(\partial\left(x^{1}+i x^{2}\right)\right) .
$$

The light-cone covariant derivatives are $D_{+}=\partial_{+}+2 i \bar{\theta}^{+}\left(\partial / \partial x^{+}\right), \bar{D}_{+}=-\bar{\partial}_{+}$, where $\partial_{+}=\partial / \partial \theta^{+}$and $\bar{\partial}_{+}=\partial / \partial \bar{\theta}^{+}$. They satisfy the algebra $\left\{D_{+}, \bar{D}_{+}\right\}=$ $-2 i\left(\partial / \partial x^{+}\right)$. We may finally make a shift in the $\bar{\phi}$ superfield. We consider

$$
\lambda\left(x_{L}^{m}, \bar{\theta}^{+}\right)=\bar{\phi}\left(x_{L}^{+}-2 i \theta^{+} \bar{\theta}^{+}, x_{L}^{-}, x_{L}^{i}, \bar{\theta}^{+}\right) .
$$

We obtain

$$
V\left(x^{m}, \theta^{\alpha}, \bar{\theta}^{\alpha}\right)=\theta^{-} \bar{\theta}^{+} \phi\left(x_{L}^{m}, \theta^{+}\right)+\theta^{+} \bar{\theta}^{-} \lambda\left(x_{L}^{m}, \bar{\theta}^{+}\right)+\theta^{-} \bar{\theta}^{-} U\left(x_{L}^{m}, \theta^{+}, \bar{\theta}^{+}\right),
$$

where $\phi$ and $\lambda$ are light-cone chiral superfields $\bar{D}_{+} \phi=0, D_{+} \lambda=0$. The expressions for $W_{+}$and $W_{-}$in terms of the covariant derivatives are

$$
\begin{aligned}
W_{+}= & -\bar{D}_{+} \lambda-\theta^{-}\left(\bar{D}_{+} D_{+} U_{+}+2 i \partial \phi\right)+g\left(\theta^{-}\left[\bar{D}_{+}\left(\bar{\theta}^{+} \lambda\right), \phi\right]\right. \\
& \left.-\theta^{-} \theta^{+}\left[D_{+} \phi, \bar{D}_{+}\left(\bar{\theta}^{+} \lambda\right)\right]-\theta^{-} \bar{\theta}^{+} \theta^{+}\left[\bar{D}_{+} D_{+} \phi, \lambda\right]\right),
\end{aligned}
$$

and

$$
\begin{aligned}
W_{-}= & -\bar{D}_{+} U+2 i \theta^{+} \bar{\partial} \lambda-2 i \bar{\theta}^{+} \theta^{+} \bar{D}_{+} \bar{\partial} \lambda+\theta^{-}\left(2 i \bar{\partial} U+2 i \bar{\theta}^{+} \bar{D}_{+} \bar{\partial} U-2 i \frac{\partial}{\partial x^{-}} \phi\right) \\
& +g\left(\theta^{-}\left[\bar{D}_{+}\left(\bar{\theta}^{+} U\right), \phi\right]-\theta^{+}\left[\phi, \bar{D}_{+}\left(\bar{\theta}^{+} \lambda\right)\right]-\theta^{-}\left[\phi, \bar{D}_{+}\left(\bar{\theta}^{+} U\right)\right]\right) .
\end{aligned}
$$

From (13a) and (8) we obtain

$$
\bar{D}_{+} \lambda=0,
$$

hence

$$
\lambda\left(x_{L}{ }^{m}, \bar{\theta}^{+}\right)=\lambda\left(x^{-}{ }_{L}, x_{L}^{i}\right)
$$


The usual assumption in SYM is that $\left(\partial / \partial x^{+}\right) \lambda=0$ implies $\lambda=0$ by means of a boundary condition. We thus have $\lambda=0$.

Equation (13a) then yields

$$
\bar{D}_{+} D_{+} U+2 i \partial \phi=0 \text {. }
$$

From (13b) and (8) we obtain

$$
\begin{gathered}
\bar{D}_{+} U=0, \\
\bar{\partial} U-\frac{\partial}{\partial x^{-}} \phi-i g[\phi, U]=0 .
\end{gathered}
$$

After using $\left\{\bar{D}_{+}, D_{+}\right\}=-2 i\left(\partial / \partial x^{+}\right),(16)$ reduces to

$$
\frac{\partial}{\partial x^{+}} U=\partial \phi
$$

Equations (17), (18) and (19) are the self dual SYM equations.

The left member of $(18)$ has the structure of a curvature in the $\left(x^{1}-i x^{2}\right)$ and $x^{-}$ directions. Taking into account (17) we get

$$
\begin{aligned}
& \phi=J^{-1} \bar{\partial} J, \\
& U=J^{-} \frac{\partial}{\partial x^{-}} J,
\end{aligned}
$$

where

$$
J\left(x_{L}^{m}, \theta^{+}\right)=j\left(x_{L}^{m}\right)+\theta^{+} \psi\left(x_{L}^{m}\right)
$$

is a light-cone chiral superfield.

From (19) we obtain

$$
\frac{\partial}{\partial x^{+}}\left(J^{-1} \frac{\partial}{\partial x^{-}} J\right)=\partial\left(J^{-1} \delta J\right) .
$$

This is the light-cone supersymmetric generalization of the $J$-formulation [4] of SDYM.

Equation (20) corresponds to the left $J$-formulation. The right $J$-formulation is, by similar arguments

$$
\begin{gathered}
\phi=J \partial^{-} J^{-1} \\
U=J \frac{\partial}{\partial x^{-}} J^{-1} \\
\frac{\partial}{\partial x^{+}}\left(J \frac{\partial}{\partial x^{-}} J^{-1}\right)=\partial\left(J \bar{\partial} J^{-1}\right) .
\end{gathered}
$$

The component equations can be directly evaluated. From (22) we obtain (using $\left.J^{-1}=j^{-1}-\theta^{+} j^{-1} \psi j^{-1}\right)$,

$$
\partial_{+}\left(j^{-1} \partial_{-} j\right)=\partial\left(j^{-1} \bar{\partial} j\right)
$$


and

$$
\partial_{+}\left(j^{-1} \partial_{-} \psi-j^{-1} \psi j^{-1} \partial_{-} j\right)=\partial\left(j^{-1} \bar{\partial} \psi-j^{-1} \psi j^{-1} \bar{\partial}\right) .
$$

Equation (24a) is the usual self dual equation in the $J$-formulation when we rotate $x^{0} \rightarrow i x^{0}$. Equation (24a) represents the evolution equation of the two bosonic degrees of freedom

$$
\square j=-j \partial_{+} j^{-1} \cdot \partial_{-} j+j \partial j^{-1} \cdot \bar{\partial} j,
$$

while $(24 b)$ is the evolution equation for the two fermionic degrees of freedom

$$
\square \psi=-j \partial_{+} j^{-1} \cdot \partial_{-} \psi+j \partial_{+}\left(j^{-1} \psi\right) \cdot j^{-1} \partial_{-} j+j \partial j^{-1} \cdot \bar{\psi} \psi-j \partial\left(j^{-1} \psi\right) j^{-1} \bar{\partial} j .
$$

We may now extend (21), (22) to $N=2$ and 4.

From (12) we know the equation for the spin 1 component field. We now find a light-cone supersymmetric equation for an $N=2$ or 4 light-cone superfield $J\left(x, \theta^{+i}\right)$ whose $\theta^{+i}=0$ component, $j(x)$, satisfies the $\theta=\bar{\theta}=0$ equation of (12) [22].

The superfields are now

$$
\begin{aligned}
J\left(x, \theta^{+i}\right)= & j(x)+\theta^{+i} \psi_{i}(x)+\theta^{+i} \theta^{+i} \phi(x), \quad N=2, \\
J\left(x, \theta^{+i}\right)= & j(x)+\theta^{+i} \psi_{i}(x)+\left(\theta^{+} \theta^{+}\right)^{[i j]} \phi_{[i j]}+\left(\theta^{+} \theta^{+} \theta^{+} \varepsilon\right)^{l} \chi_{l} \\
& +\theta^{+} \theta^{+} \theta^{+} \theta^{+} \varepsilon C(x), \quad N=4 .
\end{aligned}
$$

The only light-cone supersymmetric equation which satisfies the above requirement is again (22). Hence (22) is the field equation for the independent degrees of freedom for the self-dual SYM theories for all $N$. In addition we have found explicitly the superpotentials in terms of $J\left(x, \theta^{+}\right)$for the $N=1$ theory.

We note that we have not given a complete proof for $N=2$ and 4 that $J\left(x, \theta^{+i}\right)$ satisfying (12) gives a Lorentz-covariant theory. This was ensured for $N=1$ by our method of solving the constraints. We have not done that for $N=2$ and 4 due to their considerable complexity; we would need to do that or something equivalent to it to fully justify our claim for $J\left(x, \theta^{+i}\right)$.

\section{Section 4. Reduction to Lower Dimensional Systems}

Following [3] we wish to dimensionally reduce the self-dual SYM equation (23) to the supersymmetric analogue of various well known nonlinear systems in two dimensions.

First let us consider the reduction to the Sine-Gordon equation. We take the superpotentials dependent only on 2 coordinates, $x^{+}$and $x^{-}$. Making the same ansatz as in [3], but now in terms of light cone superfields

$$
\begin{aligned}
& A_{-}\left(x^{+}, x^{-}, \theta\right)=-\frac{i}{4} \frac{\partial}{\partial x^{-}} \phi \sigma^{3}, \quad A_{+}\left(x^{+}, x^{-}, \theta\right)=\frac{i}{4} \frac{\partial}{\partial x^{+}} \phi \sigma^{3}, \\
& A_{\tilde{y}}\left(x^{+}, x^{-}, \theta\right)=\frac{i}{2}\left(\begin{array}{cc}
0 & e^{-i \phi / 2} \\
e^{+i \phi / 2} & 0
\end{array}\right), \quad A_{y}\left(x^{+}, x^{-}, \theta\right)=\frac{i}{2}\left(\begin{array}{cc}
0 & e^{i \phi / 2} \\
e^{-i \phi / 2} & 0
\end{array}\right)
\end{aligned}
$$


with $y=x_{1}+i x_{2}$ and $\bar{y}=x_{1}-i x_{2}$, we get, after solving (23),

$$
\partial_{+} \partial_{-} \phi\left(x^{+}, x^{-}, \theta\right)=-\sin \phi\left(x^{+}, x^{-}, \theta\right) .
$$

In terms of the component fields of $\phi=B+\theta^{+} \lambda$ we obtain

$$
\begin{gathered}
\partial_{+} \partial_{-} B=-\sin B, \\
\partial_{-} \partial_{+} \lambda=-B^{-1} \sin B \cdot \lambda
\end{gathered}
$$

Equation (26) allows us now to obtain directly the generalization of the associated linear problem, and the corresponding Kac-Moody algebra. We analyse these problems in the next section.

The same approach allows a reduction to a SUSY version of the Liouville system,

$$
\left.\partial_{+} \partial_{-} \cdot w\left(x_{+}, x_{-}, \theta^{+}\right)=e^{2 w\left(x_{+}, x_{-}, \theta^{+}\right.}\right) .
$$

Another possible reduction is the SUSY chiral models in 2 dimensions. We require the superfield $J$ to be a function of $x^{+}, x^{-}$and the odd grassmannian variables.

The final reduction that we look at is the reduction to a SUSY generalization of the Ernst equation of General Relativity.

Taking the same ansatz as in the non-supersymmetric case $[29,3]$ we obtain

$$
\partial_{\rho}\left(J^{-1} \partial_{\rho} J\right)+\frac{1}{\rho}\left(J^{-1} \partial_{\rho} J\right)+\partial_{\xi}\left(J^{-1} \partial_{\xi} J\right)=0
$$

$J$ being a light-cone 2 dimensional superfield.

We give a detailed analysis of this system in [26]. In particular we discuss a supersymmetric extension of the Geroch group and of the known Bäcklund transformations.

The components of the $J$ superfield in (23) are complex fields. However in the reduction procedure from 4 to 2 bosonic dimensions we may use a reality condition on the superfield based on a Majorana condition on the two dimensional spinors.

We wish to compare the above two dimensional light-cone supersymmetric models with some of the known [30] supersymmetric models.

We do it explicitly for the Liouville system. Analogous results follow for the relation between the other models.

The action for the SUSY Liouville model [28] is

$$
I=\int d^{2} x d \theta_{1} d \theta_{2}\left(-\frac{1}{2} i \Phi \bar{D} D \Phi+e^{\Phi}\right),
$$

where

$$
\Phi=\phi+i \theta_{+} \psi_{+}+i \theta_{-} \psi_{-}+i \theta_{+} \theta_{-} F \equiv \Omega\left(x, \theta_{+}\right)+\theta_{-} \chi\left(x, \theta_{+}\right) .
$$

$\Omega\left(x, \theta_{+}\right)$and $\chi\left(x, \theta_{+}\right)$are now light-cone superfields.

The light-cone algebra is given only in terms of

$$
D_{+}=\frac{\partial}{\partial \theta_{+}}+i \theta_{+} \partial_{+}, \quad\left\{D_{+}, D_{+}\right\}=2 i \partial_{+} .
$$

The transformation law for $\Omega\left(x, \theta^{+}\right)$and its components under a SUSY 
transformation are

$$
\delta \Omega=\varepsilon^{+} D_{+} \Omega, \quad \delta \phi=i \varepsilon^{+} \psi_{+} \quad \delta \psi_{+}=\varepsilon^{+} \partial_{+} \phi .
$$

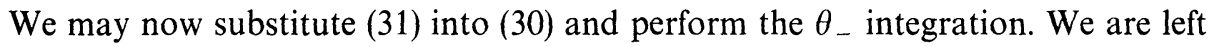
with the following light-cone superfield action

$$
I=\int d^{2} x d \theta_{+}\left\{\frac{1}{2} \Omega D_{+} \partial_{-} \Omega-\frac{1}{2} i \chi D_{+} \chi+e^{\Omega} \chi\right\} .
$$

The field equations are the known ones [28] rewritten in terms of $\Omega$ and $\chi$,

$$
\begin{gathered}
D_{+} \partial_{-} \Omega=-e^{\Omega} \chi, \\
i D_{+} \chi=e^{\Omega} .
\end{gathered}
$$

$\chi$ is an auxiliary superfield. It can be evaluated explicitly in terms of $\Omega$. From (33b) $\chi=-\partial_{+}^{-1}\left(D_{+} \Omega \cdot e^{\Omega}\right)$. The independent propagating components are thus members of a light-cone superfield $\Omega$, and the symmetry between these components is only a light cone SUSY. This is the well-known relation between the off-shell SUSY of the action and the light-cone SUSY of the propagating degrees of freedom.

In our reduction procedure (28) we end directly with a non-linear system in terms of the light-cone propagating modes. The action for the SUSY non-linear model we have is

$$
I=\int d^{2} x d \theta_{+}\left(-\omega \partial_{+} \partial_{-} \omega+e^{2 \omega}\right)
$$

The field equation is

$$
\partial_{+} \partial_{-} \omega=e^{2 \omega}
$$

In terms of components

$$
\begin{gathered}
\omega=\phi+\theta_{+} \psi, \quad \partial_{+} \partial_{-} \phi=e^{2 \phi}, \\
\partial_{+} \partial_{-} \psi=e^{2 \phi} \psi
\end{gathered}
$$

Equations (33) and (35) both describe propagating spin 0 and spin $\frac{1}{2}$ fields with the same propagating kernel, $\partial_{+} \partial_{-}$, in terms of light-cone superfields $\Omega$ and $\omega$. The explicit self interaction terms are different; however, the systems may still be related by a field redefinition, though we have not been able to determine this.

The known Bäcklund transformations for the non-SUSY Liouville model are easily generalizable. As an example we consider

$$
\begin{aligned}
& \frac{\partial \omega^{\prime}}{\partial x_{+}^{\prime}}=\frac{\partial \omega}{\partial x_{+}}+e^{1 / 2\left(\omega+\omega^{\prime}\right)}, \\
& \frac{\partial \omega^{\prime}}{\partial x_{-}^{\prime}}=-\frac{\partial \omega}{\partial x_{-}}-2 e^{1 / 2\left(\omega-\omega^{\prime}\right)},
\end{aligned}
$$

which yield as integrability conditions

$$
\partial_{+} \partial_{-} \omega=e^{2 \omega}
$$

or

$$
\partial_{+} \partial_{-} \omega^{\prime}=0
$$


From (38b) and (37) we obtain the general solution of (38a)

$$
\omega=\frac{1}{2} \ln \left[\frac{\partial_{+} F \partial_{-} G}{(F+G)^{2}}\right]
$$

where

$$
\begin{aligned}
& F=F\left(x^{+}, \theta^{+}\right)=f\left(x^{+}\right)+\theta^{+} \chi_{+}\left(x^{+}\right), \\
& G=G\left(x^{-}, \theta^{+}\right)=g\left(x^{-}\right)+\theta^{+} \chi_{-}\left(x^{-}\right)
\end{aligned}
$$

In components

$$
\omega=\frac{1}{2} \ln \left[\frac{\partial_{+} f \partial_{-} g}{(f+g)^{2}}\right]+\theta_{+}\left[\frac{\partial_{+} \chi_{+}}{\partial_{+} f}+\frac{\partial_{-} \chi_{-}}{\partial_{-} g}-2 \frac{\left(\chi_{+}+\chi_{-}\right)}{(f+g)}\right] .
$$

The bosonic component is the known solution of (36a), while the fermionic component is the new solution for its SUSY companion (36b).

\section{Section 5. The Kac-Moody Algebra}

We may follow the known approaches to obtain the hidden symmetries of the nonlinear system: the $H$-transformation method or the Riemann-Hilbert transform method $[9,27]$.

In both cases the generalization is straightforward. We discuss here briefly the first method.

The SUSY SDYM equations (22) can be obtained as integrability conditions for the linear problem

$$
\begin{gathered}
\partial \chi+\lambda \partial-\chi+\lambda\left(J^{-1} \partial_{-} J\right) \chi=0 \\
\partial_{+} \chi+\lambda \bar{\partial} \chi+\lambda\left(J^{-1} \bar{\partial} J\right) \chi=0 .
\end{gathered}
$$

It has the same structure as in the non-SUSY case [12]. $\chi$ is a Lie algebra valued light-cone superfield and $\lambda$ is an even grassmannian parameter. There is also a linear problem associated with the right $J$-formulation (23), in terms of a $\hat{\chi}$ superfield.

We consider now the following parametric transformations for the $J$-superfield,

$$
\begin{aligned}
\delta_{\alpha}(\lambda) J & =-J \chi(\lambda) T_{\alpha} \chi^{-1}(\lambda), \\
\hat{\delta}_{\alpha}\left(-\lambda^{-1}\right) J & =\hat{\chi}\left(-\lambda^{-1}\right) T_{\alpha} \hat{\chi}^{-1}\left(-\lambda^{-1}\right) J,
\end{aligned}
$$

$T_{\alpha}=\alpha^{a} T_{a}, T_{a}$ are the generators of the Lie algebra, $C_{a b}^{c}$ the structure constants. As in the non-SUSY case $J+\delta_{\alpha}(\lambda) J$ and $J+\hat{\delta}_{\alpha} J$ are solutions of (22) and (23) respectively.

From (41a) and (41b) [12] it follows that

$$
\begin{aligned}
& {\left[\delta_{\alpha}(\lambda), \delta_{\beta}\left(\lambda^{\prime}\right)\right] J=-\alpha_{a} \beta_{b} C_{a b}^{c} \frac{\left[\lambda \delta_{c}(\lambda)-\lambda^{\prime} \delta_{c}\left(\lambda^{\prime}\right)\right] J}{\lambda-\lambda^{\prime}},} \\
& {\left[\hat{\delta}_{\alpha}(\lambda), \hat{\delta}_{\beta}\left(\lambda^{\prime}\right)\right] J=-\alpha_{a} \beta_{b} C_{a b}^{c} \frac{\left[\lambda^{\prime} \hat{\delta}_{c}(\lambda)-\lambda \hat{\delta}_{c}\left(\lambda^{\prime}\right)\right] J}{\lambda-\lambda^{\prime}},} \\
& {\left[\delta_{\alpha}(\lambda), \hat{\delta}_{\beta}\left(\lambda^{\prime}\right)\right] J=-\alpha_{a} \beta_{b} C_{a b}^{c} \frac{\lambda\left[\delta_{c}(\lambda)+\hat{\delta}_{c}\left(\lambda^{\prime}\right)\right] J}{\lambda-\lambda^{\prime}} .}
\end{aligned}
$$


Expanding now in $\lambda$ one gets the commutation relations for the Kac-Moody algebra:

$$
\left[Q_{a}^{(m)}, Q_{b}^{(n)}\right]=C_{a b}^{c} Q_{c}^{(m+n)},
$$

where now

$$
\begin{aligned}
Q_{a}^{(m)} & \equiv \int d^{4} x d \theta^{N} \operatorname{tr}\left[\Delta_{a}^{(m)} J(x, \theta) \delta / \delta J(x, \theta)\right] \\
\Delta_{a}^{\left({ }^{(k)}\right.} & \equiv \delta_{a}^{(k)}, \quad k>0 \\
& \equiv \delta_{a}^{(0)}+\hat{\delta}_{a}^{(0)}, \quad k=0 \\
& \equiv \hat{\delta}_{a}^{(-k)}(-1)^{k}, \quad k \leqq 0
\end{aligned}
$$

\section{Conclusions}

We have carefully analysed S.S.D.Y.M. and its reduction in terms of physical fields only, using a manifest light-cone supersymmetric approach. We leave for further analysis the problem of solvability of non-linear systems following the geometrical approach of Atiyah. The main problem still to be solved is the $N=4 \mathrm{~S}$.Y.M. which we will be considering elsewhere, as well as the supersymmetric extensions of monopole theories. In particular the extensions of Bogomolny equations seem to arise directly from the SSD problem we have considered here.

\section{Appendix (Notation)}

The USP(2) metric is taken as

$$
\varepsilon^{i j}=-\varepsilon^{j i}=\varepsilon_{j i} \quad \varepsilon^{12}=1 \quad i, j=1,2 .
$$

The USP(4) metric is taken as

$$
\alpha^{i j}=\left(\begin{array}{rr}
0 & 1 \\
-I & 0
\end{array}\right) \text {, where } I=\left(\begin{array}{ll}
1 & 0 \\
0 & 1
\end{array}\right)
$$

$\left(x^{a}, \theta^{\underline{\alpha}} \theta^{\dot{\alpha}}\right)$ are the coordinates of the supermanifold.

$x^{a}$ are the even space-time coordinates,

$$
(a=0,1,2,3) .
$$

$\theta^{\underline{\alpha}}$ are the odd spinorial coordinates, where the underlined indices $\underline{\alpha}, \underline{\dot{\alpha}}$ stand for double indices $\left(\begin{array}{l}\alpha \\ i\end{array}\right),(\dot{\alpha} j)$ respectively, with $\alpha, \dot{\alpha}=1,2$ and $i, j=1,2, \ldots \bar{N}$. All spinor variables $\theta_{\underline{\alpha}}$ are Grassmann-Valued, with $\left[\theta_{\underline{\alpha}}, \theta_{\underline{\beta}}\right]_{+}=\left[\theta_{\underline{\alpha}}, \theta_{\underline{\underline{\beta}}}\right]_{+}=0$.

In $N=1$ supersymmetry the supercurvature tensor $F_{A B}$ is defined as the (anti) commutator of the covariant derivatives

$$
\mathscr{D}_{A}=D_{A}+\mathscr{A}_{A},
$$

where $D_{A}=\left(\partial_{a}, D_{\alpha}, \bar{D}_{\dot{\alpha}}\right)$ with $D_{\alpha}=\partial / \partial \theta^{\alpha}+i \theta^{\beta} \sigma_{\alpha \beta}^{a} \partial_{a}$ and $\bar{D}_{\dot{\alpha}}=-\partial / \partial \bar{\theta}^{\dot{\alpha}}-i \theta^{\beta} \sigma_{\beta 2}^{a} \partial_{a}$, and where $\mathscr{A}_{A}$ are the Yang-Mills superpotentials. There are similar expressions in the $N=2$ and 4 cases.

For $N=1$, the chiral superfields $W^{\alpha}, \bar{W}^{\dot{\alpha}}$ are given in terms of the supercurvature 
tensor by

$$
\bar{W}^{\dot{\alpha}}=\frac{i}{4}\left(\sigma^{a}\right)^{\dot{\alpha} \beta} F_{a \beta}, \quad W^{\alpha}=\frac{i}{4} F_{a \dot{\alpha}}\left(\sigma^{a}\right)^{\dot{\alpha} \alpha} .
$$

For $N=2$ the field strengths are defined by the superfields $W, \bar{W}$,

$$
W=\frac{g^{i j} \varepsilon^{\dot{\alpha} \dot{\beta}}}{4} \bar{F}_{\dot{\alpha} i \dot{\beta} j}, \quad \bar{W}=\frac{g_{i j} \varepsilon^{\alpha \beta}}{4} F_{\alpha \beta}^{i j} .
$$

For $N=4$ there are the superfields $W_{i j}, \bar{W}^{i j}$,

$$
W_{i j}=-\frac{1}{2} \varepsilon^{\alpha \beta} \bar{F}_{\alpha i \beta j}, \quad \bar{W}^{i j}=-\frac{1}{2} \varepsilon^{\alpha \beta} F_{\alpha \beta}^{i j} .
$$

The Bianchi identities in superspace are a generalization of the usual cyclic identity on the curvature

$$
\mathscr{D}_{A} F_{A C}+(-)^{a(b+c)} \mathscr{D}_{B} F_{C A}+(-)^{c(b+a)} \mathscr{D}_{C} F_{A B}=0 \text {. }
$$

This can be broken down into component equations; again a similar procedure can be followed for $N=2$ and 4 cases.

Acknowledgement. A. R. would like to acknowledge SERC of Great Britian for financial support while this work was being performed.

\section{References}

1. (a) Bäcklund transformations, the inverse scattering method, solutions and their applications. Miura, R. M., (ed.), Lecture Notes in Mathematics, Berlin, Heidelberg, New York: SpringerVerlag (1974)

(b) Proceedings of "Group theoretical methods in physics", Istanbul, Turkey, Serdaroglu, M., Inönü E. (eds.), Lecture Notes in Physics Vol. 180, Berlin, Heidelberg, New York: Springer 1982

(c) Proceedings of "Nonlinear Phenomenon", Oaxtepec, Mexico, K. Wolf (ed.), Lecture Notes in Physics Vol. 189, Berlin, Heidelberg, New York: Springer 1982

2. Atiyah, M.: Geometry of Yang-Mills fields (Scvola Normale Superiore, Pisa)(1979) Ward, R., Ansätz for self-dual Yang-Mills fields. Commun. Math. Phys. 80, 563 (1981)

3. Ward, R.: Integrable and solvable systems and relations among them. Preprint U. of Durham. 1985

4. Yang, C. N.: Condition of self-duality for SU (2) gauge fields on euclidean four-dimensional space. Phys. Rev. Lett. 38, 1377 (1977)

5. Brihaye, Y., Fairlie, D.B., Nuyts, J., Yates, R.: Properties of the self-dual equations for an SU (n) gauge theory, J. Math. Phys. 19, 2528 (1978)

6. Chau, L.-L.: Self dual Yang-Mills as a totally integrable system. in [1] (C)

7. Zakharov, V., Manakov, S., Novikov, S., Pitoveski, L.: Theory of solutions Moscow 1980: Bullough, R., Dodd, R (eds.): Solitons Berlin, Heidelberg, New York: Springer 1980

8. Volovich, I.: Supersymmetric Yang-Mills equations as an inverse scattering problem. Lett. Math. Phys. 7, 517 (1983):

Vladimirov, V., Volovich, I.: Preprint IC 84/128

9. Devehand, C.: An infinite number of continuity equations and hidden symmetries in supersymmetric gauge theories. Nucl. Phys. B238, 333 (1984); Chau, L.-L.: Supersymmetric Yang-Mills fields as an integrable system and connections with other non-linear systems. In: Vertex operators in mathematics and physics, Lepowsky, J., Mandelstam, S., Singer, I. (eds.), Berlin, Heidelberg, New York: Springer, 1985

10. Semikhatov, A.: The supersymmetric instanton. Phys. Lett. 120B, 171 (1983) 
11. Volovich, I.: Super-selfduality for supersymmetric Yang-Mills theory. Phys. Lett. 123B, 329 (1983)

12. Chau, L.-L., Ge, M.-L., Sinha, A., Wu, Y.-S.: Hidden-symmetry algebra for the self-dual YangMills equation. Phys. Lett. 121B, 391 (1983)

13. Osterwalder, K., Schrader, R.: Euclidean fermi fields and a Feynmen-Kac formula for bosonfermion models. Helv. Phys. Acta. 46, 277 (1973)

14. Martin, I., Taylor, J. C.: Euclidean supersymmetry (in preparation)

15. Ferrara, S., Zumino, B.: Supergauge invariant Yang-Mills theories. Nucl. Phys. B79, 413 (1974); Salam, A., Strathdee, J.: Super-symmetry and non-abelian gauges, Phys. Lett. 51B, 353 (1974)

16. Grimm, R., Sohnius M. Wess, J.: Extended supersymmetries and gauge theories. Nucl. Phys. B133, 275 (1978)

17. Sohnius, M.: Bianchi identities for supersymmetric gauge theories. Nucl. Phys. B136, 461 (1978)

18. Sohnius, M.: Supersymmetry and central charges. Nucl. Phys. B138, 109 (1978); Rands, B., Taylor, J. G.: Off-shell representations of supersymmetry with central charges. J. Phys. A.: Math. Gen. 16 1005 (1983) and Superspin classification for supersymmetry representations with unresrticed central charges, 16, 3919 (1983); Taylor, J. G.: A superfield formulation of extended supersymmetric gauge theories. Phys. Lett. 94B, 174 (1980)

19. Sohnius, M., Stelle, K., West, P.: Representations of Extended Supersymmetry. In: Superspace and Supergravity. Hawking. S., Rocek, M. (eds.), Cambridge: University Press 1981

20. Restuccia, A., Taylor, J. G.: How to integrate over central charges. J. Phys. A16, 4097 (1983)

21. Brink, L., Lindgren, O., Nilsson, B.: $N=4$ Yang-Mills theory on the light cone. Nucl. Phys. B212, 401 (1983); Hassoun, J., Restuccian, A., Taylor, J. G.: The reduction of $N=1$ supersymmetric Yang-Mills theory to the light-cone gauge, Phys. Lett. B124, 197 (1984)

22. This is the superfieldization approach used. In: Taylor, J. G.: Extended supergravity in light cone superspace, Proc. Karpacz School on Supersymmetry and Supergravity. Singapore: World Scientific

23. Card, C., Davis, P., Restuccia, A. Taylor, J. G.: On $N=4$ super Yang-Mills in $N=4$ superspace. Phys. Lett. B146, 199 (1984); On the quantization of centrally-charged $N=4$ super Yang-Mills, B151, 235 (1985); and Nuovo Cimento (to appear)

24. LuKierski, J., Nowicki, A.: Superspinors and graded Lorentz groups in three, four and five dimensions. Fortschr. Phys. 30, 75 (1982)

25. Zumino, B.: preprint TH-2327 CERN 1977

26. Gilson, C., Martin, L., Restuccia, A., Taylor, J. G.: in preparation

27. Wu, Y. S., Ge, M. L.: in Vertex operators in mathematics and physics, (eds.), Lepowsky, J., Mandelstam, S., Singer, I. Berlin, Heidelberg, New York: Springer, 1985

28. Chaichain, M., Kulish, F.: On the method of inverse scattering problem and Bäcklund transformations for supersymmetric equations. Phys. Lett. B78, 413 (1978)

29. Kinnersley, W., Chitre, D. M.: Symmetries of the stationary Einstein-Maxwell equations. IV. Transformations which preserve asymptotic flatness. J. Math. Phys. 19, 2037 (1978); Kinnersley, W.: Symmetries of the stationary Einstein-Maxwell field equations. I. J. Math. Phys. 18, 1529 (1977); Hauser, I., Ernst, J. F.: Integral equation method for effecting Kinnersley-Chitre transformations, Phys. Rev. D20, 362 (1979)

30. Di Vecchia, P., Ferrara, S.: Classical solutions in two-dimensional supersymmetric field theories. Nucl. Phys. B130, 93 (1977); Choudhury, A. Roy: ICTP preprint/83/101 (1983); Din, A., Lukierski, J., Zakrzewski, W.: General classical solutions of a supersymmetric non-linear coupled boson-fermion model in two dimensions. Nucl. Phys. B194, 157 (1982)

Communicated by C. H. Taubes

Received March 17, 1986; in revised form June 8, 1986 
